\title{
THREE-WAVE NONLINEAR INTERACTIONS OF DISTURBANCES IN SUPERSONIC BOUNDARY LAYER ON THE SOLID AND POROUS SURFACES
}

\author{
S.A. GAPONOV , N.M.TEREKHOVA \\ Khristianovich Institute of Theoretical and Applied Mechanics SB RAS \\ Novosibirsk, 630090 \\ RUSSIA \\ gaponov@itam.nsc.ru terekh@itam.nsc.ru http://www.itam.nsc.ru
}

\begin{abstract}
The interaction of disturbances in supersonic boundary layer is considered within the framework of the weakly nonlinear stability theory for the Mach number $\mathrm{M}=2$ on the solid and porous surfaces. The interrelations in several triplets composed of two- and three-dimensional waves at the frequencies related by the phase synchronization conditions were modelled. It was found that their interactions on the solid surface are much stronger in the asymmetric triplet. It was found that on a porous surface, the linear increments of vortex disturbances increase considerably, the region of dangerous frequencies widens, and the spatial extension of the existence of growing oscillations increases. Nonlinear interactions are, as a rule, much more intense in comparison with the case of an solid surface; they realize in a broad frequency range, which results to a broadband growth of the Tollmien - Schlichting subharmonic vortex waves. An increase in the surface porosity leads to the intensification of nonlinear processes.
\end{abstract}

Key-Words: stability, transition, supersonic and hypersonic flows, nonlinear waves.

\section{Introduction}

The practical need of affecting the character of the flow and the problem of obtaining the given flow characteristics make using various active and passive techniques of controlling the boundary layers. Among them there is the use of various flexible, wavy, porous, heated and cooled surfaces, the distributed and slot aspiration and suction

In the present work a theoretical investigation of the peculiarities of the development of vortex disturbances at the linear and nonlinear stages of the evolution in supersonic boundary layer of an solid and porous surfaces has been carried out for the Mach number $M=2$. The variation of the characteristics of disturbances on a plate was considered at the linear stage for different porosity degrees, and we further simulate within the framework of the weakly nonlinear stability theory the interaction of vortex disturbances in threewave resonance systems - the triads composed of various two-and three-dimensional oscillations.

Such investigations carried out for the hypersonic regime $M>5$ showed that the main peculiarities accompanying the initial stages of the stability loss and transition are described sufficiently completely by the mathematical apparatus developed in this section of mechanics $[1,2]$ and correspond to the data of experiments [3]. All the above-said was the reason for conducting such a research in supersonic regimes at moderate Mach numbers, when only the vortex oscillations are selected in the flow. The understanding of the nature of physical processes following the nonlinear evolution of unstable disturbances in such boundary layers can, besides the important fundamental importance for the given flow type, help at the analysis of similar interactions at hypersonic velocities.

\section{Basic formulas and methods}

The basic assumptions of the nonlinear model of the interaction in three-wave resonance systems for compressible boundary layers were presented in detail in [4-6]. We present some necessary assumptions. Denote by $\varepsilon$ the fluctuation field scale $(\varepsilon<<1)$. A disturbed field of the velocities, density, pressure, and temperature of the compressible gas is considered:

$$
\begin{array}{ll}
u=U(Y)+\varepsilon u^{\prime}, \quad v=\varepsilon v^{\prime}, \quad w=\varepsilon w^{\prime}, & \rho_{0}=\rho(Y)+\varepsilon \zeta^{\prime}, \\
p_{0}=P(Y)+\varepsilon p^{\prime}, & T_{0}=T(Y)+\varepsilon \Theta^{\prime}, \quad p^{\prime} / P=\zeta / \rho+\Theta^{\prime} / T
\end{array}
$$

in a dimensionless Cartesian coordinate system $X, Y, Z$ $=(x, y, z) / \delta$, where $\delta=\left(v_{e} x / U_{e}\right)^{1 / 2}$ is the reference scale, $e$ is the index of the parameters at the external edge of the boundary layer. The quantities with primes and 
without the primes are the fluctuation and mean components of corresponding quantities. The solution is constructed with the aid of the expansion in the small parameter $\varepsilon$ and the two-scale expansion of the streamwise coordinate. Besides the "rapid" scale $X$, a "slow" scale $\xi=\varepsilon X$ is introduced, which is justified by a big difference between the velocities of the change of the phase and amplitudes of disturbances.

Wave solutions are sought in the form

$$
(\bar{Z})^{\prime}{ }_{j}=A_{j}(\xi)(\bar{Z})_{j}^{0}(Y) \exp \left(i \theta_{j}\right)+\text { c.c. }+\varepsilon \bar{Z}_{j}^{\prime 1}+\ldots j=1, \ldots, 3
$$

where $\bar{Z}^{\prime}=\left|u^{\prime}, v^{\prime}, w^{\prime}, p^{\prime}, \Theta^{\prime}\right|$ are the proper amplitude functions of the streamwise, normal, and transversal (lateral) components of wave velocities, the pressure and temperature disturbances, $A$ is the slowly varying amplitude, c.c. are the complex conjugate quantities, $\theta=\alpha X+\beta Z-\omega t, \alpha=\alpha_{r}+i \alpha_{i}, \alpha_{i}<0$ is the increment, the real frequency $\omega=2 \pi f$, the wave numbers $\alpha, \beta$ and the frequency $\omega$ are related by the dispersion relation $\alpha=\alpha(\omega, \beta)$ according to the linear theory.

From the full system of the equations of motion and conservation for compressible gas, we obtain for the vector function $\bar{Z}$ within the framework of the weakly nonlinear theory the following recurrence system for disturbances [4]:

$$
\begin{aligned}
& \varepsilon\left\{\sum_{j=1}^{n} e^{i \theta_{j}}\left[L\left(\bar{Z}_{j}^{0}\right)+\partial L\left(\bar{Z}_{j}^{0}\right) / \partial \alpha_{j} \partial / \partial X++\partial L\left(\bar{Z}_{j}^{0}\right) / \partial \omega_{j} \partial / \partial t\right] A_{j}+\right. \\
& \left.+\varepsilon \sum_{k, l}^{n} e^{i\left(\theta k+\theta_{l}\right)}\left[L\left(\bar{Z}_{k+l}^{1}\right)+A_{k} A_{l} \bar{M}_{k, l}^{j}\left(\bar{Z}_{j}^{0} \bar{Z}_{l X}^{0}, \ldots\right)\right]\right\}=0
\end{aligned}
$$

the boundary conditions for which will be considered below. The linear operator $L$ has the form (the coefficients of $F$ on the right-hand sides are equal to zero)

$$
\begin{aligned}
& {\left[\rho G u+U_{Y} v+\left(i \alpha p / \gamma \mathrm{M}^{2}\right)-(\mu / \operatorname{Re}) u_{Y Y}\right] \exp (i \theta)=-F_{u},} \\
& {\left[\rho G w+i \beta p / \gamma \mathrm{M}^{2}-\mu / \operatorname{Re} w_{Y Y}\right] \exp (i \theta)=-F_{w},} \\
& {\left[\rho G v+\left(p_{Y} / \gamma \mathrm{M}^{2}\right)\right] \exp (i \theta)=-F_{v},} \\
& G \zeta+\rho_{Y} v+\rho\left(i \alpha u+v_{Y}+i \beta w\right) \exp (i \theta)=-F_{p}, \\
& \zeta=\rho\left(\frac{p}{P}-\frac{\Theta}{T}\right), \quad G=i-\omega+\alpha U .
\end{aligned}
$$

Here $\mathrm{M}$ is the Mach number at the external boundary, $y=c_{p} / c_{v}$ is the adiabatic exponent, $\sigma=c_{p \mu e} / k$ is the Prandtl number, $k$ is the thermal conductivity coefficient, $\bar{M}$ is the sum of nonlinear terms of the governing equations.

The boundary conditions for disturbances at the solid surface have the form $u, v, w, \Theta=0(Y=0, Y=\infty)$.

Consider the boundary conditions for a porous surface. At high values of the normal coordinate $Y$, these are the conditions for disturbances decay at infinity; as in (4), on the wall $Y=0$ these are $u, w, \Theta=0$, as well as the relation $v(0)=K p(0)$ obtained from the motion equation and the permeability law $[2,7,8]$.

A number of models were proposed to construct the coefficient $K$. In the present work, we make use of the model from the works $[7,8]$. It considers a perforated plate with the diameter of cylindrical pores $\bar{r}_{1}$ and the distances between them, which are small in comparison with the boundary layer thickness. The pores are oriented along the normal to the surface. It was obtained that $K=n \cdot \operatorname{th}(\lambda H) / Z_{0}$, where $n$ is the porosity, $H$ is the porous coating thickness, $\lambda$ is the propagation constant, $Z_{0}$ is the characteristic impedance. By analogy with the electroconductive line [9], the quantities $\lambda$ and $Z_{0}$ are expressed in terms of the pipe element impedance $Z_{1}$ and the coefficient characterizing the compression energy reserve and the losses due to the heat transfer to the walls $W$. These acoustic parameters characterize the relation between the volumetric velocity being average over the pipe (pore) section and the pressure. It was obtained that in the dimensionless form [7]:

$$
Z_{1}=i \alpha c \frac{I_{0} \sqrt{i \alpha c \operatorname{Re}} r_{1}}{I_{2} \sqrt{i \alpha c \operatorname{Re}} r_{1}}, r_{1}=\bar{r}_{1} / \delta, c=\omega / \alpha^{r}, \lambda=\sqrt{Z_{1} W}, Z_{0}=\sqrt{\frac{Z_{1}}{W}} .
$$

$W=-i \alpha c \mathrm{M}^{2}\left[\gamma+(\gamma-1) \frac{I_{2} \sqrt{i \alpha c \operatorname{Re} \sigma} r_{1}}{I_{0} \sqrt{i \alpha c \operatorname{Re} \sigma} r_{1}}\right]$,

Here $I_{0}$ and $I_{2}$ are the Bessel functions of the zero and second orders.

In the first order in $\varepsilon$ the homogeneous system (3) is the basis for finding the eigenvalues $\alpha$ at given $\beta, \omega$ and Reynolds numbers $\operatorname{Re}_{x}=x / \delta\left(\mathrm{Re}=\sqrt{\operatorname{Re}_{x}}\right)$ as well as for constructing the proper amplitude functions of linear waves (1) at an indefinite $A$. In the weakly nonlinear theory, the above parameters are assumed to be sought, the nonlinearity affects only the wave amplitude $A$.

The process of paired interaction of the waves in the third wave field under the conditions of the synchronization of their phases $\theta_{j}=\theta_{k}+\theta_{l}$ underlies the resonance model. In the second order of $\varepsilon$, one can find from inhomogeneous equations (2) the higherorder disturbances $\bar{Z}^{1}$ as well as construct the amplitude equations for resonance triads by using the solvability conditions. 
$d A_{j} / d \xi=-\alpha_{j}^{i} A_{j}+S_{k, l}^{j} A_{K} A_{l} \exp (i \Delta)$,

$d A_{k} / d \xi=-\alpha_{k}^{i} A_{k}+S_{j, l}^{k} A_{j} A_{l}^{*} \exp (i \Delta)$,

$S_{k, l}^{j}=\int_{0}^{Y_{k}} \bar{Z}_{j}^{0+} \bar{M}_{k, l}^{j} d Y / \int_{0}^{Y_{k}} \bar{Z}_{j}^{0+} \partial L\left(\bar{Z}_{j}^{0}\right) / \partial \alpha_{j} d Y$,

$\Delta=\int \alpha_{k}+\alpha_{l}-\alpha_{j}{ }^{r} \Delta X$.

Consider the initial conditions for complex amplitudes $A=|A| \exp (i \psi)$. If the intensities $I$ of wave components are known in the initial section $\mathrm{Re}_{0}$, then one can express $|A|$ and $\psi$ in terms of the computed values of the mass velocity fluctuation $m: I\left(\mathrm{Re}_{0}\right)=$ $=|A| m\left(Y_{m}\right) \exp \left(-\alpha^{i} \operatorname{Re}_{0}\right)$, and $\psi$ is arbitrary, usually $\psi\left(\operatorname{Re}_{0}\right)=0$.

For each wave mode, the dimensionless frequency parameter $F$ related to the frequency by the dependency $\omega=\operatorname{Re} F$ was introduced for convenience, and also the reduced dimensionless wave number $b=\beta \cdot 10^{3} / \mathrm{Re}$ remained constant. Both the plane two-dimensional (2D) waves with $\beta=0$ and the oblique threedimensional (3D) waves with different $\beta \neq 0$ are considered in the present work.

We consider the obtained results after these necessary explanations.

\section{Results and discussion}

The attachment to the experiments was made in computations. The experiments were conducted in the boundary layer of a plate at the freestream Mach number $\mathrm{M}=2$ and the unit Reynolds number $R_{1}=6.5 \cdot 10^{6}$ $\mathrm{m}^{-1}$. The artificial modulated disturbances were introduced in the flow by a spark source so that the signals were revealed on the oscillograms of the downstream fluctuations at the harmonic frequency $\left(f_{G}=20 \mathrm{kHz}\right)$ and at the subharmonic frequency $\left(f_{S}=10 \mathrm{kHz}\right)$. In the dimensionless form, these frequencies corresponded to the frequency parameters $f_{G}=0.38 \cdot 10^{-4}$ and $f_{S}=$ $0.19 \cdot 10^{-4}$. The measurements were carried out in the range $3.5 \cdot 10^{5} \leq R_{x} \leq 5.3 \cdot 10^{5}$, which corresponds to the Reynolds numbers Re $>620$. System (5) was solved for $600 \leq \operatorname{Re} \leq 1600$. The stagnation temperature was constant in experiments and amounted to $390 \mathrm{~K}, \gamma=$ 1.4 , the Prandtl number $\sigma=0.72$. The same parameters were used at the modelling.

Several triplets connecting the disturbances at the above frequencies are considered in the work. The table presents the data enabling the representation of waves in the $x, z$ plane.

\begin{tabular}{|c|c|c|c|}
\hline $\begin{array}{l}\text { Tri- } \\
\text { plet }\end{array}$ & $\begin{array}{l}\text { Dimension } \\
\text { of the } \\
\text { harmonic on } \\
F_{G}=.38 \cdot 10^{-4}\end{array}$ & $\begin{array}{l}\text { Dimension of } \\
\text { the } 1 \mathrm{st} \text { sub- } \\
\text { harmonic on } \\
F_{S}=0.19 \cdot 10^{-4}\end{array}$ & $\begin{array}{l}\text { Dimension of } \\
\text { the 2nd sub- } \\
\text { harmonic on } \\
F_{S}=0.19 \cdot 10^{-4}\end{array}$ \\
\hline 1 & $3 \mathrm{D}(b=0.12)$ & $3 \mathrm{D}(b=0.212)$ & $3 \mathrm{D}(b=-0.092)$ \\
\hline 2 & 2D $(b$ & $3 \mathrm{D}(b=0.075)$ & $3 \mathrm{D}(b=-0.075)$ \\
\hline 3 & $3 \mathrm{D}(b=0 . \mathrm{c}$ & $3 \mathrm{D}(b=$ & $2 \mathrm{D}(b=0)$ \\
\hline 4 & $2 \mathrm{D}(b=0)$ & $2 \mathrm{D}(b=0)$ & $2 \mathrm{D}(b=$ \\
\hline
\end{tabular}

The porous surface parameters corresponded to the technologically acceptable values, namely: in the main variants of computations, the porosity $n=0.5$, the dimensionless values of the porous coating thickness $H=3 \cdot 10^{4}$ corresponded to the surfaces pasted onto the substrate in $(0.75 \div 1)$, and the pore radii $r_{1}=$ $600(0.05 \div 0.1) \mathrm{mm}$.

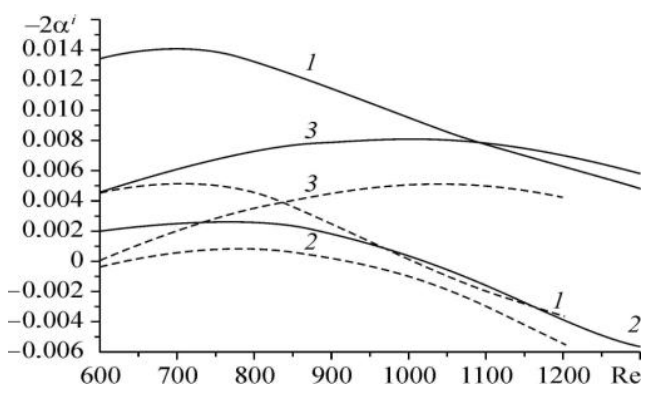

Fig. 1. Streamwise amplification rate $\alpha^{i}$ vs. the Reynolds number for linear waves of the first asymmetric triplet for the frequency parameter $F=0.38 \cdot 10^{-4}$ (l) and the subharmonics at $F=0.19 \cdot 10^{-4}$ ( 2 and 3 ) on the solid (dashed lines) and porous (solid lines) surfaces.

Figure 1 shows the most representative characteristics of vortex waves - the spatial growth rates $\alpha^{i}$ for the solid and porous surfaces (the dashed and solid lines) of the components of the first triplet: the harmonic and two subharmonics (digits 1-3). It was found that the increments of all waves on the porous surface increase, and this means that the spatial growth rates are increasing. The spatial regions of the existence of the growing evolution regions also increase considerably. If the point of the neutral curve upper branch for the harmonic on the solid surface lies in the neighborhood of $\operatorname{Re} \sim 1050$, then it shifts on the porous surface into the region $\operatorname{Re} \sim 3000$. The same trend (although not so explicitly) is observed also for subharmonic components.

The same dependencies are presented in subsequent figures for the components 2-4 of the triplets Nos. 2-4, in Fig. 2, $a$ they are shown for the two- dimensional (curves 1 ) and three-dimensional (curves 2) harmonics, and in Fig. 2, $b$ for the 2D and for the 3D 
subharmonics. The common peculiarities are here exactly the same as in the first triplet. These curves must help at the interpretation of data in nonlinear regions.
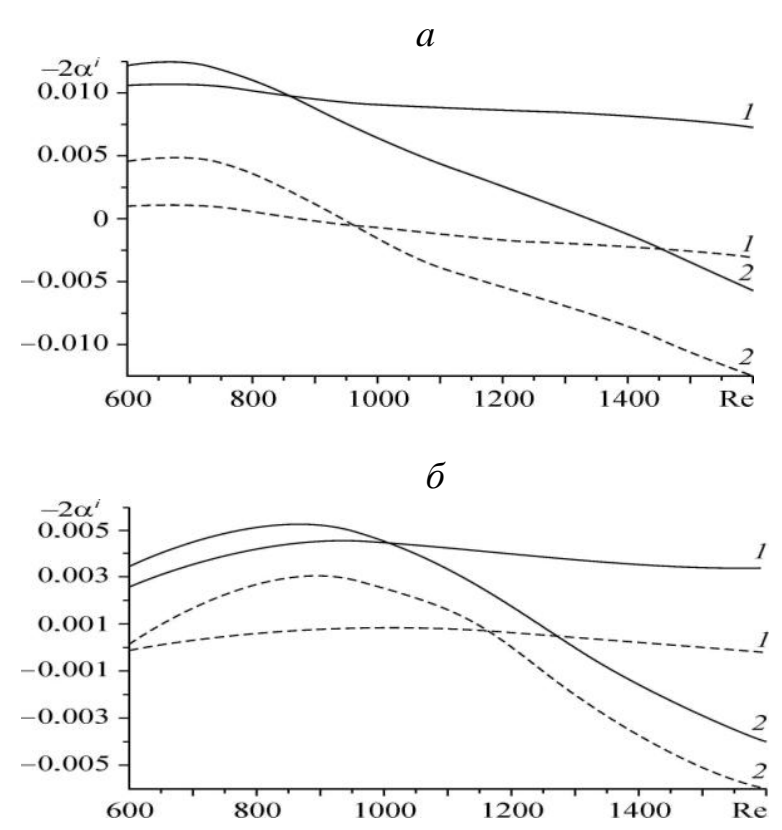

Fig. 2. Linear increments $\alpha^{i}$ vs. Re of vortex waves at the frequency of the harmonic $F=0.38 \cdot 10^{-4}(a)$ and for the subharmonics $F=0.19 \cdot 10^{-4}(b)$ on the solid (dashed lines) and porous (solid lines) surfaces.

Two-dimensional waves (1), three-dimensional waves with $b=0.075$ of symmetric triplets Nos. 2-4 (2).

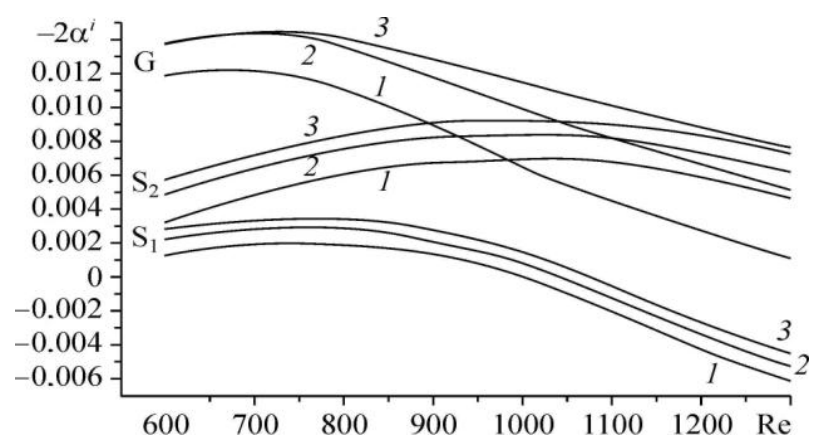

Fig. 3. The $\alpha^{i}$ vs. Re for linear waves of the triplet 1 for $n=0.25$ (1), 0.5 (2), 0.75 (3) for the harmonic $(G)$ (1), the first $S_{1}(2)$ and the second $S_{2}(3)$ subharmonics.

Figure 3 shows the influence of the porosity degree $n$ on the increments of the first triplet. The family $G$ determines the dependencies for the harmonic, the family $S_{1}$ determines the dependencies for the first subharmonic, and the family $S_{2}$ determines the dependencies for the second subharmonic. Digits 1-3 mark here the data for $n=0.25,0.5$, and 0.75 . It becomes evident that a porosity increase destabilizes more strongly the disturbances of all triplet components, and this manifests itself especially in wave parameters at a higher frequency.

One can formulate the following hypothesis on the reasons of such an influence of the porosity: because of the fact that on such a wall in the near-wall region, the normal wave component differs from zero, the force characteristics of the Reynolds stresses increase. As a result a more powerful vortex forms in the near-wall layer and the waves related to it enhance and grow more rapidly.

At the modelling, the initial intensity of the fluctuations of the mass velocity of the harmonic component was chosen predominant and equal to $0.1 \%$ of the mean value of $\rho U$. The initial intensities of subharmonic components were, as a rule, lower by an order of magnitude. Threshold values of the intensities of the onset of nonlinear interactions were different for different triplets, for example, for the first triplet, this value was $0.01 \%$ of the mean $\rho U$ for the harmonic, and for other triplets, it was much higher.

The following figures directly illustrate the nonlinear interaction in different three-wave systems. A system for data representation was chosen in the form of the ratio $I_{n} / I_{\text {lin }}$, one can immediately see in the figure the relation between the nonlinear ( $n$ ) and linear (lin) processes. Figure 4 shows the data for the first triplet. It was found that the nonlinear interaction realizes therein in the parametric pumping regime. The harmonic (digit 1) component is the pumping wave, which develops according to the linear law both on the solid (dashed) and on the porous (solid lines) surfaces.

It was found that only the first subharmonic marked by digit 2 grows nonlinearly in the given triplet. This growth starts manifesting itself already at low Re numbers and is substantial on the both surfaces. The growth rate of this component in the nonlinear region is more significant on the porous surface. One can only suppose that a preferable amplification of this subharmonic in the given triplet is related to its low linear increments. The second subharmonic (digit 3) develops according to a purely linear law.

According to system (5), the spatial evolution of the amplitudes of the triplets components is determined by both linear amplification in accordance with their increments $\alpha^{i}$ (the first terms in the right-hand sides of equations) and by nonlinear additions represented by the mutual influence coefficients $S$. One can only suppose that a preferable nonlinear amplification of the first subharmonic in the given triplet is related to its low linear increments $\alpha^{i}$, which one can clearly see in Fig. 3 (family $S_{1}$ ), and to the prevailing of the nonlinear term in this sum.

For the second subharmonic (family $S_{2}$, see Fig. $3)$, linear increments are fairly high as compared to the first one, which just determines a relative predomin- 
ance of linear amplification in the process of the streamwise evolution of this component.

An investigation of the influence of the porosity degree $n$ on nonlinear interaction was conducted for the asymmetric triplet. The values $n=0.25,0.5$, and 0.75 were considered, the increments for the given degrees are shown in Fig. 3. For this variant, the initial intensities of subharmonics were somewhat lower than in the above-considered case. Fig. 5 shows the relative intensities of the first subharmonic in the nonlinear region. As was to be expected an increase in the surface porosity intensifies the nonlinear growth of this component.

The following figures directly illustrate the nonlinear interaction in different three-wave systems. A system for data representation was chosen in the form of the ratio $I_{n} / I_{\text {lin }}$, one can immediately see in the figure the relation between the nonlinear $(n)$ and linear (lin) processes. Figure 4 shows the data for the first triplet. It was found that the nonlinear interaction realizes therein in the parametric pumping regime. The harmonic component is the pumping wave, which develops according to the linear law both on the solid (dashed) and on the porous (solid lines) surfaces.

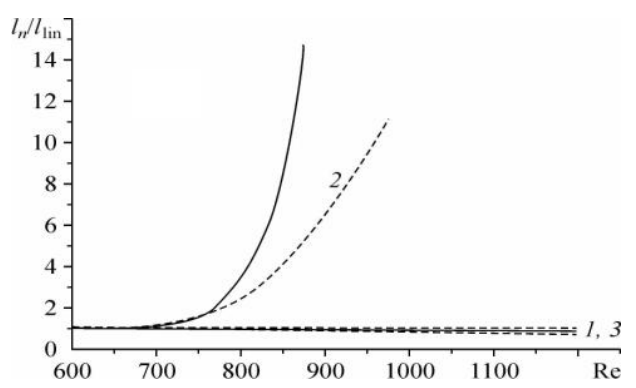

Fig. 4. The ratio of nonlinear and linear intensities $I_{n} / I_{\text {lin }}$ of the interacting waves of the triplet 1 vs. Re on the solid (dashed lines) and porous (solid lines) surfaces for the harmonic and subharmonics (1-3).

It was found that only the first subharmonic marked by digit 2 grows nonlinearly in the given triplet. This growth starts manifesting itself already at low Re numbers and is substantial on the both surfaces. The growth rate of this component in the nonlinear region is more significant on the porous surface. One can only suppose that a preferable amplification of this subharmonic in the given triplet is related to its low linear increments. The second subharmonic (digit 3) develops according to a purely linear law.

According to system (5), the spatial evolution of the amplitudes of the triplets components is determined by both linear amplification in accordance with their increments $\alpha^{i}$ (the first terms in the right-hand sides of equations) and by nonlinear additions represented by the mutual influence coefficients $S$. One can only suppose that a preferable nonlinear amplification of the first subharmonic in the given triplet is related to its

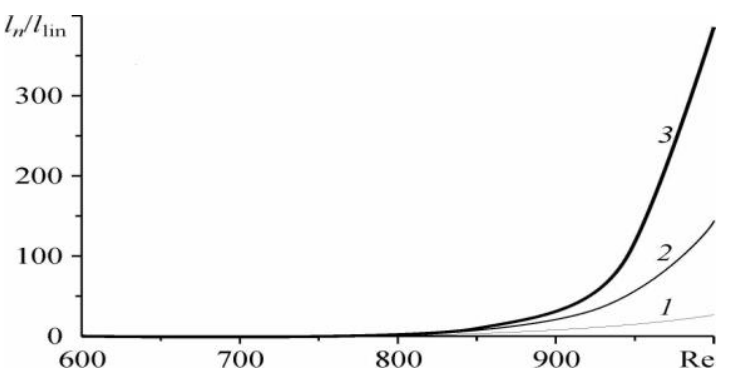

Fig. 5. The ratio of nonlinear and linear intensities $I_{n} / I_{\text {lin }}$ Vs. Re for the first subharmonic of the first triplet on the porous surface for different porosity degrees. $n$ $=0.25(1), 0.5(2), 0.75(3)$.
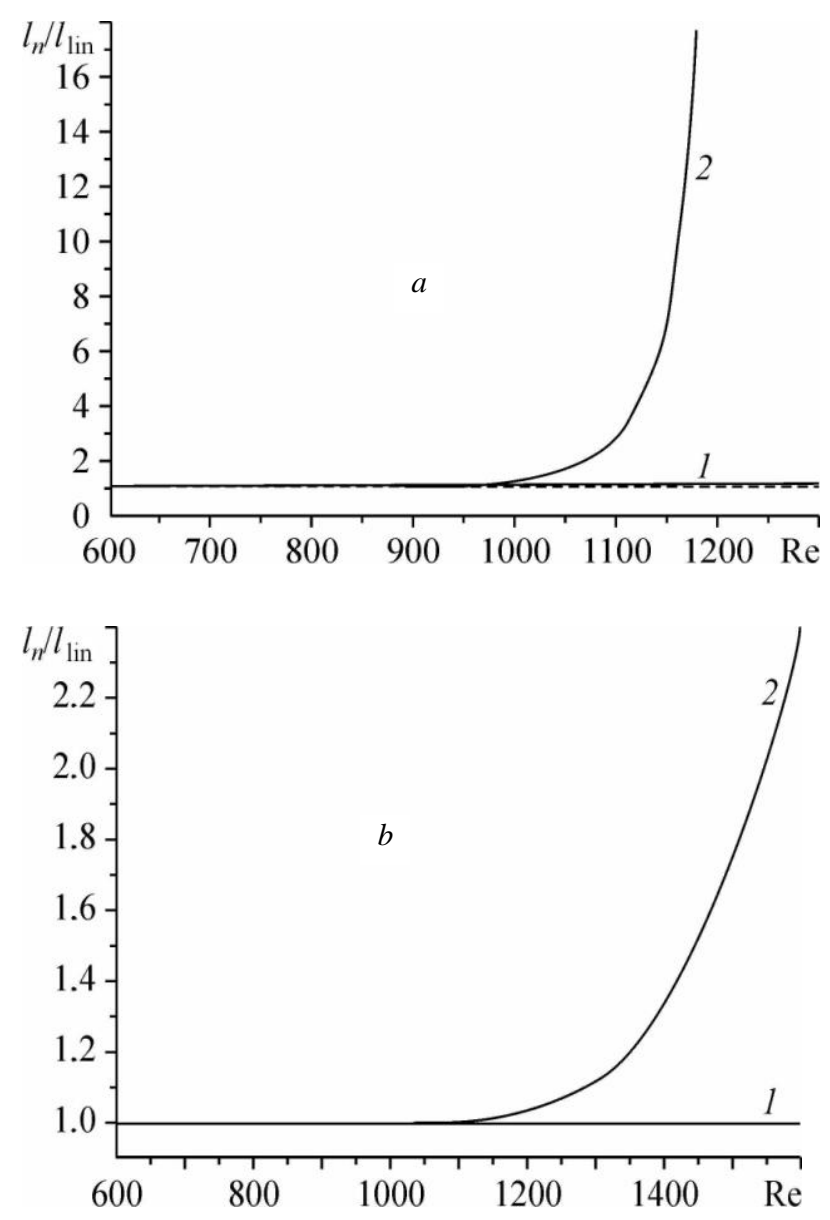

Fig. 6. The ratio of $I_{n} / I_{\text {lin }}$ vs. Re.

$a$ : for the $2 \mathrm{D}$ harmonic (1) and the 3D subharmonic (2) of the triplet 2 on a porous surface for $n=0.5$ (2) and for the solid surface (the dashed line);

$b$ : for the 2D harmonic (1) and 2D subharmonic (2) of the triplet $4(n=0.5)$;

low linear increments $\alpha^{i}$, which one can clearly see in Fig. 3 (family $S_{1}$ ), and to the prevailing of the nonlinear term in this sum.

For the second subharmonic (family $S_{2}$, see Fig. 3 ), linear increments are fairly high as compared to the first one, which just determines a relative predomin- 
ance of linear amplification in the process of the streamwise evolution of this component.

An investigation of the influence of the porosity degree $n$ on nonlinear interaction was conducted for the asymmetric triplet. The values $n=0.25,0.5$, and 0.75 were considered, the increments for the given degrees are shown in Fig. 3. For this variant, the initial intensities of subharmonics were somewhat lower than in the above-considered case. Figure 5 shows the relative intensities of the first subharmonic in the nonlinear region. As was to be expected, an increase in the surface porosity intensifies the nonlinear growth of this component.

Nonlinear interactions in triplets Nos. 2 and 4 (Fig. $6, a, b)$ proceed similarly to this scenario. Due to symmetry it is enough to watch therein the behavior of only one subharmonic (digit 2 in Fig. 6). The given initial intensity of the harmonic component on the solid surface proves to be lower than the threshold one, and purely linear processes determine the behavior of all waves. Nonlinear interactions are noticeable only on a porous surface, they realize in the same regime of the parametric pumping and lead to the amplification of subharmonic components, and the three-dimensional subharmonic (Fig. 6, a) is amplified much earlier and more strongly than the two-dimensional one (Fig. 6, $b$ ).

A comparison of the intensities of nonlinear processes in triplet 1 and triplets 2 and 4 confirms a conclusion about the priority of the asymmetric (experimental) triplet in the nonlinear region.

Nonlinear interactions in triplet 3 differ considerably from the ones considered above, and the evolution of waves therein may be termed anomalous.

\section{CONCLUSIONS}

Nonlinear interactions in supersonic boundary layers were found to realize in most cases in the parametric resonance regime. The wave at a higher frequency (the harmonic) is the pumping wave, the process passes with a preferable amplification of subharmonic components having moderate increments in the linear region. Nonlinear interactions are as a rule much more intense on the porous surface, they realize in a wide frequency range, which leads to a broadband growth of subharmonic vortex waves.

It was found that there exist the triplets in which relatively weak nonlinear interactions realize between the waves in the redistribution regime, which leads to a minor decay of the harmonic and to a slight amplification of subharmonic components.

\section{References:}

[1] S.A. Gaponov, N.M. Terekhova, and B.V. Smorodsky, Three-wave interaction of disturbances in hypersonic boundary layer, Vestnik NGU, Ser. Fizika, 2008, Vol. 3, Iss. 3, P. 39-45.

[2] S.A. Gaponov and N.M. Terekhova, Three-wave interactions between disturbances in the hypersonic boundary layer on impermeable and porous surfaces, Fluid Dyn., 2009, Vol. 44, No. 3, P. 362-371.

[3] D.A. Bountin, A.N. Shiplyuk, A.A. Maslov, and N. Chokani, Nonlinear aspects of hypersonic boundary layer stability on a porous surface, in: 42nd AIAA Aerospace Sci. Meeting and Exhibit, 2004/Reno, NV; AAIA Paper, 2004, No. 0258.

[4] S.A. Gaponov and I.I. Maslennikova, Subharmonic instability of supersonic boundary layer, Thermophysics Aeromechanics, 1997, Vol. 4, No. 1, P. $1-10$.

[5] S.A. Gaponov and N.M. Terekhova, Evolution of disturbances of elevated intensity in supersonic boundary layer, Aeromekhanika i gazovaya dinamika, 2003, No. 1, P. 28-38.

[6] A. Tumin, Nonlinear interaction of wave trains in a supersonic boundary layer, Phys. Fluids, 1996, Vol. 8, No. 9, P. 2552-2554.

[7] S.A. Gaponov, Influence of gas compressibility on the stability of a boundary layer above a permeable surface at subsonic speeds, Prikl. mekh. techn. fiz., 1975, No. 1, P. 121-125.

[8] S.A. Gaponov, Influence of the porous coating properties on the boundary layer stability, Izv. SO AN SSSR, Ser. tekhn. nauk, 1971, No. 3, Iss. 1, P. 21-23.

[9] F.B. Daniels, On the propagation of sound waves in a cylindrical conduit, J. Acoust. Soc. Amer., 1950, Vol. 22, No. 5, P. 563-564. 